\title{
Zonotopic fault detection observer design for Takagi-Sugeno fuzzy systems
}

\author{
Jitao $\operatorname{Li}^{a}$, Zhenhua Wang ${ }^{a, *}, \mathrm{Yi} \mathrm{Shen}^{a}$, Ye Wang ${ }^{b}$ \\ ${ }^{a}$ School of Astronautics, Harbin Institute of Technology, Harbin 150001, P. R. China \\ ${ }^{b}$ Institut de Robòtica i Informàtica Industrial, CSIC-UPC, Universitat Politècnica de Catalunya-BarcelonaTec \\ (UPC), C/. Llorens i Artigas 4-6, 08028, Barcelona, Spain
}

\begin{abstract}
This paper considers zonotopic fault detection observer design in the finite-frequency domain for discrete-time Takagi-Sugeno fuzzy systems with unknown-but-bounded disturbances and measurement noise. We present a novel fault detection observer structure, which is more general than the commonly used Luenberger form. To make the generated residual sensitive to faults and robust against disturbances, we develop a finite-frequency fault detection observer based on generalized Kalman-Yakubovich-Popov lemma and P-radius criterion. The design conditions are expressed in terms of linear matrix inequalities. The major merit of the proposed method is that residual evaluationa can be easily implemented via zonotopic approach. Numerical examples are conducted to demonstrate the proposed method.
\end{abstract}

Keywords: Fault detection, Takagi-Sugeno fuzzy systems, Finite-frequency domain, Observer design, Zonotopes.

\section{Introduction}

As an effect way to improve safety and reliability, model-based fault detection has been intensively studied in the past decades (Xu et al., 2014; Ren et al., 2017; Pan \& Yang, 2017). The main tasks of model-based fault detection are residual generation and residual evaluation(Li \& Yang, 2015; Basseville et al., 1993). Among the existing residual generation methods, observer

\footnotetext{
${ }^{*}$ Corresponding author. E-mail:zhenhua.wang@hit.edu.cn
} 
and filter design are predominant, see e.g. Zhuang et al. (2016); Li et al. (2018). In practice, model uncertainty, disturbance and measurement noise are inevitable. To avoid false alarms, an appropriate threshold is necessary. However, as pointed out in (Khan \& Ding, 2011), few work on residual evaluation and threshold computation has been done. So far, how to design an appropriate threshold for fault detection is still a challenge.

Based on a general assumption that the uncertainties are unknown but bounded, set-membership method has been proved to be an effective way to handle the effect of uncertainties. In the literature, different geometries are used to bound the uncertainties, e.g. ellipsoid (Kurzhanski \& Varaiya, 2000), interval (Raïssi et al., 2012) and zonotope (Wang et al., 2018). Among the existing set-membership methods, due to the flexibility, less complexity and the high computation efficiency, the zonotopic approach has been widely studied in recent years (Le et al., 2013a). In Alamo et al. (2005), segment minimization and volume minimization are used to minimize the size of the obtained zonotopes. Based on Alamo et al. (2005), Le et al. (2013b) proposes P-radius minimization which can achieve a good trade-off between segment minimization and volume minimization. Tornil-Sin et al. (2014); Xu et al. (2014); Puig (2010) extend the zonotopic approach to fault detection. The main idea behind these methods is to check the consistence between the observed system behaviour and that predicted by a fault-free model. However, the aforementioned references only consider the robustness to uncertainties, without taking the fault sensitivity into consideration.

In Wang et al. (2017a), a preliminary result by combining the zonotopic method with fault sensitivity analysis is presented. Note that Wang et al. (2017a) only considers sensor fault detection in full frequency domain, and it may have conservatism. In practice, fault often emerges in finitefrequency domain, e.g. incipient fault (Chen \& Patton, 2012). By using weighting functions, Liu et al. (2005) first extends the fault sensivity $H_{-}$index into finite-frequency case. However, it is 
time-consuming to search for approximate weighting functions (Wang \& Yang, 2008). Fortunately, the generalized Kalman-Yakubovich-Popov (GKYP) lemma proposed in Iwasaki \& Hara (2005) provides an effective way to handle the design problem in finite-frequency domain. Based on the GKYP lemma, many researches about fault detection in finite-frequency domain have been reported in recent years (Zhou et al., 2017; Wang et al., 2017b).

Many practical systems are nonlinear systems and the established linear system theory can not be applied directly. Fortunately, Takagi-Sugeno (T-S) fuzzy system provides an effective way to describe a class of nonlinear systems and is a bridge between the linear system theory and nonlinear systems (Tanaka \& Wang, 2004). As a result, control and observer design based on T-S fuzzy systems have attracted much attention, see e.g. Zhang et al. (2015); Chang et al. (2014); Zhai et al. (2018) and the reference therein. Chibani et al. (2016) proposes a finite-frequency unknown input observer design method for T-S fuzzy systems. This method is further extended to fault detection in Chibani et al. (2017). Chadli \& Karimi (2013) proposes an unknown input observer design method to decouple the effect of unknown inputs for T-S fuzzy systems. However, restrictive rank conditions are required. Youssef et al. (2017) considers a proportional intergral observer to reconstruct actuator and sensor faults for T-S fuzzy systems. However, Youssef et al. (2017) only considers the point estimation, which is less representative when the uncertainties are large. Unlike Chadli \& Karimi (2013) and Youssef et al. (2017), we propagate unknown inputs using zonotopic set-membership method. Consequently, the restrictive rank conditions can be relaxed and the generated zonotopes can be used as admissible sets of states.

Note that the existing results on fault detection based on set-membership method only consider the disturbance robustness, without considering the fault sensitivity. To the best of our knowledge, only Wang et al. (2017a) considers fault sensitivity in zonotopic observer design. However, Wang et al. (2017a) uses Luenberger observer form for linear system and does not take the finite-frequency 
characteristics of fault into consideration, which may lead to conservatism. In view of this, we develop a zonotopic fault detection observer design method for T-S fuzzy systems in finite-frequency domain. The main contributions of this paper are three-fold:

- We present a novel observer structure for discrete-time T-S fuzzy systems. Compared with conventional Luenberger form in Xu et al. (2014); Wang et al. (2017a), the merit of the proposed observer is that it can reduce conservatism and enhance performance by providing more design parameters.

- Note that conventional set-membership fault detection methods, e.g. Zhang \& Yang (2017); Xu et al. (2014); Puig (2010), only consider disturbance robustness, without considering fault sensitivity. To improve fault detection performance, we consider fault sensitivity by introducing finite-frequency $H_{-}$index.

- Note that conventional finite-frequency fault detection methods, e.g. Wang et al. (2017b); Chen et al. (2015); Zhou et al. (2017), focus on residual generation, extra residual evaluation functions are needed. By applying zonotopic approach, the proposed method can achieve residual generation and residual evaluation simultaneously.

The remainder of this paper is organized as follows. Problem statement and some preliminaries about zonotope are given in Section 2. Based on P-radius minimization and GKYP lemma, the main results on zonotopic fault detection observer design in finite-frequency domain are presented in Section 3. Numerical simulations are conducted in Section 4 to demonstrate the effectiveness of the proposed method. Finally, the conclusions are given in Section 5.

\section{Problem statement and preliminaries}

\subsection{Preliminaries}

In the following, we introduce some notations and definitions which will be used in this paper. 
Throughout this paper, we use the following notations: $\mathbb{R}^{n}$ and $\mathbb{R}^{n \times m}$ denote the $n$ dimensional Euclidean space and the set of $n \times m$ real matrices, respectively. $j$ denotes the imaginary unit, $I$ denotes the identity matrix with compatible dimensions, $\|\cdot\|$ represents the $\mathscr{L}_{2}$-norm. For a matrix $A, A^{T}$ stands for its transposition, $A^{*}$ stands for its complex conjugate transpose, $A^{\perp}$ stands for its orthogonal complement, and $\operatorname{He}(A)$ is used to denote $\operatorname{He}(A):=A+A^{T}$. For a symmetric matrix $P, P>0(P<0)$ indicates that $P$ is positive definite (negative definite). An asterisk $\star$ is used to represent a term induced by symmetry.

Definition 2.1: A zonotope $\mathcal{Z} \in \mathbb{R}^{n}$ is defined as follows

$$
\mathcal{Z}=\langle p, H\rangle=\left\{p+H b, b \in \mathbf{B}^{m}\right\},
$$

where $p \in \mathbb{R}^{n}$ is the center of the zonotope, $\mathbf{B}=[-1,1]$ is the unit interval, and $H \in \mathbb{R}^{n \times m}$ is the generator matrix of zonotope $\mathcal{Z}$.

Definition 2.2 (Le et al., 2013b): The $P$-radius of a zonotope $\mathcal{Z}$ is defined as

$$
R_{P}=\max _{z \in \mathcal{Z}}\|z-p\|_{P}^{2}=\max _{z \in \mathcal{Z}}(z-p)^{T} P(z-p),
$$

where $P=P^{T}>0$ is a symmetric and positive definite matrix.

Definition 2.3: The Minkowski sum $\oplus$ and linear product $\odot$ in zonotope operation are defined as follows:

$$
\begin{aligned}
\left\langle p_{1}, H_{1}\right\rangle \oplus\left\langle p_{2}, H_{2}\right\rangle & =\left\langle p_{1}+p_{2},\left[H_{1}, H_{2}\right]\right\rangle, \\
L \odot\langle p, H\rangle & =\langle L p, L H\rangle .
\end{aligned}
$$

The following lemmas are used in this paper.

Lemma 2.1 (Finsler's Lemma) (Boyd et al., 1994): Letting $x \in \mathbb{R}^{n}, \mathscr{L} \in \mathbb{R}^{n \times n}$ and $\mathscr{U} \in \mathbb{R}^{n \times m}$, then the following statements are equivalent:

(i) $x^{T} \mathscr{L} x<0, \quad \forall x \neq 0, \mathscr{U}^{\perp} x=0$;

(ii) $\mathscr{U}^{\perp} \mathscr{L}\left(\mathscr{U}^{\perp}\right)^{T}<0$; 
(iii) $\exists \mathscr{Y} \in \mathbb{R}^{m \times n}$ such that $\mathscr{L}+\mathscr{U} \mathscr{Y}+\mathscr{Y}^{T} \mathscr{U}^{T}<0$;

where $\mathscr{U}^{\perp}$ is any matrix that satisfies $\mathscr{U}^{\perp} \mathscr{U}=0$.

Lemma 2.2 (Wang et al., 2014): Given matrices $\mathcal{B}$ and $\mathcal{Y}$, there exists a matrix $\mathcal{X}$ such that $\mathcal{X B}=\mathcal{Y}$ if and only if

$$
\operatorname{rank}\left[\begin{array}{c}
\mathcal{B} \\
\mathcal{Y}
\end{array}\right]=\operatorname{rank}(\mathcal{Y})
$$

Moreover, the general solution to $\mathcal{X} \mathcal{B}=\mathcal{Y}$ is given by

$$
\mathcal{X}=\mathcal{Y} \mathcal{B}^{\dagger}+\mathcal{S}\left(I-\mathcal{B B}^{\dagger}\right)
$$

where $\mathcal{S}$ is an arbitrary matrix.

\subsection{System description}

In this paper, we consider T-S fuzzy system, which is described by IF-THEN rules as follows:

Rule $i$ : IF $\xi_{1 k}$ is $\mathcal{M}_{i 1}, \cdots$ and $\xi_{g k}$ is $\mathcal{M}_{i g}$, THEN

$$
\left\{\begin{array}{l}
x_{k+1}=A_{i} x_{k}+B_{i} u_{k}+F_{i} f_{k}+D_{i} w_{k} \\
y_{k}=C x_{k}+E v_{k}
\end{array}, \quad i=1,2, \cdots, s,\right.
$$

where $x_{k} \in \mathbb{R}^{n_{x}}$ is the state vector, $y_{k} \in \mathbb{R}^{n_{y}}$ is the output vector, $u_{k} \in \mathbb{R}^{n_{u}}$ is the input vector, $f_{k}$ is the actuator fault vector, $w_{k} \in \mathbb{R}^{n_{w}}$ denotes the process disturbance and $v_{k} \in \mathbb{R}^{n_{v}}$ denotes the measurement noise. $A_{i} \in \mathbb{R}^{n_{x} \times n_{x}}, B_{i} \in \mathbb{R}^{n_{x} \times n_{u}}, F_{i} \in \mathbb{R}^{n_{x} \times n_{f}}, D_{i} \in \mathbb{R}^{n_{x} \times n_{w}}, C \in \mathbb{R}^{n_{y} \times n_{x}}$ and $E \in \mathbb{R}^{n_{y} \times n_{v}}$ are known constant matrices, $s$ is the number of IF-THEN rules, $\xi_{1_{k}}, \cdots, \xi_{g k}$ are measurable premise varibles and $\mathcal{M}_{i 1}, \cdots, \mathcal{M}_{i g}$ are the fuzzy sets.

The overall fuzzy system can be described as

$$
\left\{\begin{array}{l}
x_{k+1}=\sum_{i=1}^{s} h_{i}\left(\xi_{k}\right)\left\{A_{i} x_{k}+B_{i} u_{k}+F_{i} f_{k}+D_{i} w_{k}\right\} \\
y_{k}=C x_{k}+E v_{k}
\end{array}\right.
$$

where

$$
\left\{\begin{array}{l}
\xi_{k}=\left[\xi_{1 k}, \xi_{2_{k}}, \cdots, \xi_{g k}\right]^{T}, \\
h_{i}\left(\xi_{k}\right)=\frac{\zeta_{i}\left(\xi_{k}\right)}{\sum_{i=1}^{s} \zeta_{i}\left(\xi_{k}\right)}, \\
\zeta_{i}\left(\xi_{k}\right)=\prod_{j=1}^{\prod_{i j}\left(\xi_{j k}\right) .}
\end{array}\right.
$$


Herein, the term $M_{i j}\left(\xi_{j k}\right)$ represents the grade of membership of $\xi_{j_{k}}$ in $\mathcal{M}_{i j}$. Without loss of generality, it is assumed that $\zeta_{i}\left(\xi_{k}\right) \geq 0, i=1,2 \cdots, s$. Then, the weighting functions $h_{i}\left(\xi_{k}\right), i=$ $1, \cdots, s$ satisfy:

$$
\left\{\begin{array}{l}
\sum_{i=1}^{s} h_{i}\left(\xi_{k}\right)=1 \\
h_{i}\left(\xi_{k}\right) \geq 0, \quad i=1, \cdots, s .
\end{array}\right.
$$

For the sake of brevity, we rewrite system (2) as follows:

$$
\left\{\begin{array}{l}
x_{k+1}=A(h) x_{k}+B(h) u_{k}+F(h) f_{k}+D(h) w_{k}, \\
y_{k}=C x_{k}+E v_{k}
\end{array}\right.
$$

where

$$
\begin{aligned}
& A(h)=\sum_{i=1}^{s} h_{i}\left(\xi_{k}\right) A_{i}, B(h)=\sum_{i=1}^{s} h_{i}\left(\xi_{k}\right) B_{i}, \\
& F(h)=\sum_{i=1}^{s} h_{i}\left(\xi_{k}\right) F_{i}, D(h)=\sum_{i=1}^{s} h_{i}\left(\xi_{k}\right) D_{i} .
\end{aligned}
$$

In this paper, we assume that the process disturbance, measurement noise and initial state are all unknown but bounded as follows:

$$
w_{k} \in \mathcal{W}=\left\langle 0, H_{w}\right\rangle, v_{k} \in \mathcal{V}=\left\langle 0, H_{v}\right\rangle, x(0) \in \mathcal{X}_{0}=\left\langle p_{0}, H_{0}\right\rangle
$$

where $p_{0}$ denotes the center of zonotope $\mathcal{X}_{0}, H_{w}, H_{v}$ and $H_{0}$ are the corresponding known generator matrices of zonotopes.

Remark 2.1: To simplify the computation, $\mathcal{W}$ and $\mathcal{V}$ are assumed to be centered at the origin. Note that, if this assumption is not satisfied, a change of coordinates can be used.

\subsection{Problem statement}

The main task of this paper is to design a zonotopic observer in finite-frequency domain. First, motivated by Wang et al. (2012) and Wang et al. (2015), we construct the following fault detection observer:

$$
\left\{\begin{array}{l}
\chi_{k+1}=T A(h) \hat{x}_{k}+T B(h) u_{k}+L(h)\left(y_{k}-C \hat{x}_{k}\right) \\
\hat{x}_{k}=\chi_{k}+N y_{k} \\
r_{k}=y_{k}-C \hat{x}_{k}
\end{array}\right.
$$


where $\hat{x}_{k} \in \mathbb{R}^{n_{x}}$ is the state estimation vector, $\chi_{k} \in \mathbb{R}^{n_{x}}$ is intermediate variable, $r_{k} \in \mathbb{R}^{n_{y}}$ is the residual vector. $T \in \mathbb{R}^{n_{x} \times n_{x}}, N \in \mathbb{R}^{n_{x} \times n_{y}}$ and $L(h) \in \mathbb{R}^{n_{y} \times n_{x}}$ are the matrices to be designed. $L(h)$ has the following form:

$$
L(h)=\sum_{i=1}^{s} h_{i}\left(\xi_{k}\right) L_{i}
$$

Besides, $T$ and $N$ should be designed to satisfy the following equation:

$$
T+N C=I_{n_{x}}
$$

According to Lemma 2.2, the general solution to (10) is

$$
\begin{aligned}
& T=\bar{C} \eta_{1}+S\left(I_{n_{x}+n_{y}}-\bar{C} \bar{C}^{\dagger}\right) \eta_{1}, \\
& N=\bar{C} \eta_{2}+S\left(I_{n_{x}+n_{y}}-\bar{C} \bar{C}^{\dagger}\right) \eta_{2},
\end{aligned}
$$

where $S \in \mathbb{R}^{n_{x} \times\left(n_{x}+n_{y}\right)}$ is a predetermined matrix and matrices $\bar{C} \in \mathbb{R}^{\left(n_{x}+n_{y}\right) \times n_{x}}, \eta_{1} \in \mathbb{R}^{\left(n_{x}+n_{y}\right) \times n_{x}}$ and $\eta_{2} \in \mathbb{R}^{\left(n_{x}+n_{y}\right) \times n_{y}}$ are

$$
\bar{C}=\left[\begin{array}{c}
I_{n_{x}} \\
C
\end{array}\right]^{\dagger}, \eta_{1}=\left[\begin{array}{c}
I_{n_{x}} \\
0
\end{array}\right], \eta_{2}=\left[\begin{array}{c}
0 \\
I_{n_{y}}
\end{array}\right]
$$

Remark 2.2: Note that if we choose $T=I_{n_{x}}$ and $N=0$, observer (8) reduces to the commonly used Luenberger form, e.g. in Xu et al. (2014); Wang et al. (2017a). It is obvious that the proposed structure can provide more design degrees of freedom by introducing matrices $T$ and $N$.

To analyze and synthesize the observer (8), we define the following state estimation error:

$$
e_{k}=x_{k}-\hat{x}_{k}
$$

Subtracting (8) from (5), we obtain the following error dynamic system:

$$
\left\{\begin{array}{l}
e_{k+1}=[T A(h)-L(h) C] e_{k}+T F(h) f_{k}+T D(h) w_{k}-L(h) E v_{k}-N E v_{k+1} \\
r_{k}=C e_{k}+E v_{k} .
\end{array}\right.
$$


Inspired by Wang et al. (2017b), we split error dynamic system (14) into three subsystems as follows:

$$
\begin{aligned}
\left\{\begin{array}{l}
e_{d_{k+1}}= \\
r_{d_{k}}=C e_{d_{k}}+E v_{k},
\end{array}\right. & \left\{\begin{array}{l}
e_{f_{k+1}}=[T A(h)-L(h) C] e_{f_{k}}+T F(h) \bar{f}_{k}, \\
r_{f_{k}}=C e_{f_{k}},
\end{array}\right. \\
& \left\{\begin{array}{l}
\tilde{e}_{f_{k+1}}=[T A(h)-L(h) C] \tilde{e}_{f_{k}}+T F(h) \tilde{f}_{k}, \\
\tilde{r}_{f_{k}}=C \tilde{e}_{f_{k}},
\end{array}\right.
\end{aligned}
$$

where

$$
e_{k}=e_{f_{k}}+e_{d_{k}}+\tilde{e}_{f_{k}},
$$

with $e_{f}(0)=0, e_{d}(0)=e(0), \tilde{e}_{f}(0)=0$ and $f_{k}$ is split as

$$
f_{k}=\bar{f}_{k}+\tilde{f}_{k}
$$

such that

$$
\mathrm{e}^{j \vartheta_{w}} \sum_{k=0}^{\infty}\left(e_{f_{k+1}}-\mathrm{e}^{j \vartheta_{1}} e_{f_{k}}\right)\left(e_{f_{k+1}}-\mathrm{e}^{j \vartheta_{2}} e_{f_{k}}\right)^{*} \leq 0,
$$

where $\vartheta_{w}=\left(\vartheta_{2}-\vartheta_{1}\right) / 2, \vartheta_{1}$ and $\vartheta_{2}$ are known scalars, which describe the interested frequency range in fault sensitivity analysis.

Remark 2.3: According to Iwasaki et al. (2005), inequality (18) is the time-domain interpretation of frequency range $\vartheta_{1} \sim \vartheta_{2}$. In other words, $e_{f_{k}}$ belongs to the interested frequency range $\vartheta_{1} \sim \vartheta_{2}$. Similar statement can be found in Ding \& Yang (2010) and Wang et al. (2017b).

Motivated by the definitions given in Wang et al. (2017b), we define the finite-frequency $H_{-}$ index for the purpose of fault detection as follows.

Definition 2.4: The error system in (14) is said to have a finite-frequency $H_{-}$index $\beta$, if its subsystem (16) satisfies the following inequality

$$
\sum_{k=0}^{\infty} r_{f_{k}}^{T} r_{f_{k}} \geq \beta^{2} \sum_{k=0}^{\infty} \bar{f}_{k}^{T} \bar{f}_{k}
$$


where $\bar{f}_{k}$ is a part of $f_{k}$ such that (18) holds.

Remark 2.4: The reason for splitting the error dynamic system (14) to three subsystems are two-fold. First, the initial condition of fault subsystem is zero. Thus, Definition 2.4 does not require the zero initial condition assumption, which is necessary in Ding \& Yang (2010) and Li \& Yang (2014). Second, $e_{k}$ may not belong to the specified finite-frequency domain. In view of this, we split error dynamic system (14) into three subsystems such that $e_{f_{k}}$ in fault subsystem (16) belongs to finite-frequency range.

On the other hand, the effect of uncertainties on residual is described by the size of zonotopes.

According to (5) and (10), we have

$$
\begin{aligned}
x_{k+1} & =(T+N C) x_{k+1}+L(h)\left(y_{k}-C x_{k}-E v_{k}\right) \\
& =(T+N C)\left[A(h) x_{k}+B(h) u_{k}+F(h) f_{k}+D(h) w_{k}\right]+L(h)\left(y_{k}-C x_{k}-E v_{k}\right) \\
& =[T A(h)-L(h) C] x_{k}+T B(h) u_{k}+T F(h) f_{k}+L(h) y_{k}+N y_{k+1}+T D(h) w_{k} \\
& -L(h) E v_{k}-N E v_{k+1} .
\end{aligned}
$$

Considering the state $x_{k}$ in (5) is bounded by a zonotope $x_{k} \in \mathcal{X}_{k}=\left\langle p_{k}, H_{k}\right\rangle$, then, according to (7) and (20), we have

$$
\begin{aligned}
x_{k+1} & \in \mathcal{X}_{k+1}=\left\langle p_{k+1}, H_{k+1}\right\rangle \\
& =[T A(h)-L(h) C] \odot\left\langle p_{k}, H_{k}\right\rangle \oplus T B(h) u_{k} \oplus T F(h) f_{k} \oplus L(h) y_{k} \oplus N y_{k+1} \\
& \oplus T D(h) \odot\left\langle 0, H_{w}\right\rangle \oplus L(h) E \odot\left\langle 0, H_{v}\right\rangle \oplus N E \odot\left\langle 0, H_{v}\right\rangle .
\end{aligned}
$$

Using the zonotopic operation defined in Definition 2.3, the center $p_{k+1}$ and the generated matrix $H_{k+1}$ of $\mathcal{X}_{k+1}$ are calculated as

$$
\begin{aligned}
& p_{k+1}=T A(h) p_{k}+T B(h) u_{k}+T F(h) f_{k}+L(h)\left(y_{k}-C p_{k}\right)+N y_{k+1}, \\
& H_{k+1}=\left[(T A(h)-L(h) C) \downarrow_{l}\left(H_{k}\right) \quad T D(h) H_{w} \quad L E H_{v} \quad N E H_{v}\right] .
\end{aligned}
$$


Then, the corresponding residual zonotope $r_{k+1} \in \mathcal{R}_{k+1}=\left\langle p_{k+1}^{r}, H_{k+1}^{r}\right\rangle$ has the following form:

$$
p_{k+1}^{r}=y_{k+1}-C p_{k+1}, H_{k+1}^{r}=C H_{k+1} .
$$

Substituting (22) into (23), (23) yields

$$
\begin{aligned}
& p_{k+1}^{r}=(I-C N) y_{k+1}-C L(h) y_{k}-(C T A(h)-C L(h) C) p_{k}-C T B(h) u_{k}-C T F(h) f_{k}, \\
& H_{k+1}^{r}=\left[C(T A(h)-L(h) C) \downarrow_{l}\left(H_{k}\right) \quad C T D(h) H_{w} \quad C L E H_{v} \quad C N E H_{v}\right] .
\end{aligned}
$$

Remark 2.5: Note that the orders of zonotope $\mathcal{X}_{k}$ and $\mathcal{R}_{k}$ increase at each integration step, the computation load becomes quickly prohibitive. Thus, to reduce the computational load, the reduced operator $\downarrow_{l}(\cdot)$ from Combastel (2015) is adopted. For more details, please refer to Combastel (2015).

We now present the zonotopic fault detection observer design problem, as follows.

Zonotopic fault detection observer design : Given the finite frequency ranges (i.e. $\vartheta_{1}$ and $\vartheta_{2}$ ), we aim to design fault detection observer (8) for T-S fuzzy system (5) such that

(i) The error system (15) is asymptotically stable.

(ii) The zonotopic observer in (8) is robust to uncertainties, more specifically, the $P$-radius of zonotopes generated by (14) is minimized.

(iii) The error system in (14) has a finite-frequency $H_{-}$index $\beta$.

To facilitate the observer design, the following lemma is presented and will be used in the later development.

Lemma 2.3 : Assuming that system (16) is stable and given a constant $\beta>0$, system (16) has a finite-frequency performance $\beta$, if there exist matrices $P(h)=P(h)^{T}$ and $Q=Q^{T}>0$ for any $h:=\left(h_{1}(\xi(k)), \cdots, h_{s}(\xi(k))\right), h^{+}:=\left(h_{1}(\xi(k+1)), \cdots, h_{s}(\xi(k+1))\right)$ such that

$$
\left[\begin{array}{cc}
T A(h)-L(h) C & T F(h) \\
I & 0
\end{array}\right]^{T} \Xi\left[\begin{array}{cc}
T A(h)-L(h) C & T F(h) \\
I & 0
\end{array}\right]+\left[\begin{array}{cc}
C & 0 \\
0 & I
\end{array}\right]^{T} \Pi\left[\begin{array}{cc}
C & 0 \\
0 & I
\end{array}\right] \leq 0,
$$


where

$$
\Pi=\left[\begin{array}{cc}
-I & 0 \\
0 & \beta^{2} I
\end{array}\right]
$$

and

(i) for the low-frequency range $|\theta| \leq \vartheta_{l}$, we have

$$
\Xi=\left[\begin{array}{cc}
-P\left(h^{+}\right) & Q \\
Q & P(h)-2 \cos \vartheta_{1} \mathrm{Q}
\end{array}\right],
$$

(ii) for the middle-frequency range $\vartheta_{1} \leq \theta \leq \vartheta_{2}$, we have

$$
\Xi=\left[\begin{array}{cc}
-P\left(h^{+}\right) & \mathrm{e}^{j \vartheta_{c}} Q \\
\mathrm{e}^{-j \vartheta_{c}} Q & P(h)-2 \cos \vartheta_{w} Q
\end{array}\right],
$$

where

$$
\vartheta_{c}=\left(\vartheta_{1}+\vartheta_{2}\right) / 2, \quad \vartheta_{w}=\left(\vartheta_{2}-\vartheta_{1}\right) / 2,
$$

(iii) for the high-frequency range $|\theta| \geq \vartheta_{h}$, we have

$$
\Xi=\left[\begin{array}{cc}
-P\left(h^{+}\right) & -Q \\
-Q & P(h)-2 \cos \vartheta_{h} Q
\end{array}\right] .
$$

Proof: First, we consider the middle-frequency case. Multiplying the inequality $(25)$ by $\left[e_{f_{k}}^{T}, \bar{f}_{k}^{T}\right]$ from the left and by its transpose from the right, we have

$$
\begin{aligned}
e_{f_{k}}^{T} P(h) e_{f_{k}}-e_{f_{k+1}}^{T} P\left(h^{+}\right) e_{f_{k+1}} & +\beta^{2} \bar{f}_{k}^{T} \bar{f}_{k}-r_{f_{k}}^{T} r_{f_{k}} \\
& +\operatorname{tr}\left(Q\left(\mathrm{e}^{j \vartheta_{c}} e_{f_{k}} e_{f_{k+1}^{T}}+\mathrm{e}^{-j \vartheta_{c}} e_{f_{k+1}} e_{f_{k}^{T}}-2 \cos \vartheta_{w} e_{f_{k}} e_{f_{k}}^{T}\right)\right) \leq 0 .
\end{aligned}
$$

Taking the summation from $k=0$ to $\infty$, in view of $e_{f}(0)=0$ and $\lim _{k \rightarrow \infty} e_{f_{k}}=0$ (due to stability of (16)), we have

$$
\beta^{2} \sum_{k=0}^{\infty} \bar{f}_{k}^{T} \bar{f}_{k}-\sum_{k=0}^{\infty} r_{f_{k}}^{T} r_{f_{k}}+\operatorname{tr}(Q S) \leq 0
$$

where

$$
S:=\sum_{k=0}^{\infty}\left(\mathrm{e}^{j \vartheta_{c}} e_{f_{k}} e_{f_{k+1}}^{T}+\mathrm{e}^{-j \vartheta_{c}} e_{f_{k+1}} e_{f_{k}}^{T}-2 \cos \vartheta_{w} e_{f_{k}} e_{f_{k}}^{T}\right)
$$


It can readily verify that $S$ is equivalent to the negative of the left-hand side of (18), and thus, $S$ is positive semidefinite. Since $Q>0$, the second term on the left-hand side of (30) is non-negative when (18) is satisfied. Hence, (19) is satisfied. Similarly, the results follow by choosing $\vartheta_{2}:=\vartheta_{l}$ and $\vartheta_{1}:=-\vartheta_{l}$ for the low-frequency case and $\vartheta_{2}:=2 \pi-\vartheta_{h}$ and $\vartheta_{1}:=-\vartheta_{h}$ for the high-frequency case.

Remark 2.6: Note that if we set $Q=0$ in Lemma 2.3, we will get the result in the full-frequency domain.

\section{Main results}

\subsection{Zonotopic fault detection observer design}

In this subsection, we present a design method for zonotopic fault detection observer (8). Based on GKYP lemma and P-radius minimization method in Le et al. (2013b), we present the following theorem:

Theorem 3.1: For given scalars $\gamma>0$, the designed zonotopic observer (8) is robust against disturbance and has a finite-frequency performance $\beta$, if there exist a scalar $\kappa>0$, matrices $Q=Q^{T}>0 \in \mathbb{R}^{n_{x} \times n_{x}}, P_{f i}=P_{f i}^{T} \in \mathbb{R}^{n_{x} \times n_{x}}, P_{d i}=P_{d i}^{T}>0 \in \mathbb{R}^{n_{x} \times n_{x}}, G \in \mathbb{R}^{n_{x} \times n_{x}}, W_{i} \in \mathbb{R}^{n_{x} \times n_{y}}$ and $Y \in \mathbb{R}^{n_{x} \times\left(n_{x}+n_{y}\right)}$ for all $i, j=1,2 \cdots, s$ such that

$$
\begin{gathered}
P_{d i} \geq \kappa I_{n_{x}}, \\
{\left[\begin{array}{cccc}
\tilde{v}_{11} & \star & \star \\
\tilde{v}_{21} & \tilde{v}_{22} & & \star \\
\tilde{v}_{31} & \tilde{v}_{32} & -P_{f j}-G-G^{T}
\end{array}\right]<0,} \\
{\left[\begin{array}{ccccc}
\tilde{\lambda}_{11} & \star & \star & \star & \star \\
\tilde{\lambda}_{21} & \tilde{\lambda}_{22} & \star & \star & \star \\
\tilde{\lambda}_{31} & 0 & \tilde{\lambda}_{33} & \star & \star \\
\tilde{\lambda}_{41} & 0 & 0 & \tilde{\lambda}_{44} & \star \\
\tilde{\lambda}_{51} & \tilde{\lambda}_{52} & \tilde{\lambda}_{53} & \tilde{\lambda}_{54} & P_{d j}-G-G^{T}
\end{array}\right]<0,}
\end{gathered}
$$


where

$$
\begin{aligned}
& \tilde{v}_{11}=P_{f i}-2 \cos \vartheta_{w} Q-C^{T} C+\mathbf{H e}\left\{\alpha_{1}\left(G \bar{C} \eta_{1} A_{i}+Y\left(I-\bar{C} \bar{C}^{\dagger}\right) \eta_{1} A_{i}-W_{i} C\right)\right\}, \\
& \tilde{v}_{21}=V\left(G \bar{C} \eta_{1} A_{i}+Y\left(I-\bar{C} \bar{C}^{\dagger}\right) \eta_{1} A_{i}-W_{i} C\right)+\left(\alpha_{1} G \bar{C} \eta_{1} F_{i}+Y\left(I-\bar{C} \bar{C}^{\dagger}\right) \eta_{1} F_{i}\right)^{T}, \\
& \tilde{v}_{22}=\beta^{2} I+\mathbf{H e}\left\{V G \bar{C} \eta_{1} F_{i}+Y\left(I-\bar{C} \bar{C}^{\dagger}\right) \eta_{1} F_{i}\right\}, \\
& \tilde{v}_{31}=\mathrm{e}^{j \vartheta_{c}} Q-\alpha_{1} G^{T}+G \bar{C} \eta_{1} A_{i}+Y\left(I-\bar{C} \bar{C}^{\dagger}\right) \eta_{1} A_{i}-W_{i} C, \\
& \tilde{v}_{32}=-G^{T} V^{T}+G \bar{C} \eta_{1} F_{i}+Y\left(I-\bar{C} \bar{C}^{\dagger}\right) \eta_{1} F_{i}, \\
& \tilde{\lambda}_{11}=-\gamma P_{d i}+\mathbf{H e}\left\{\alpha_{2}\left[G \bar{C} \eta_{1} A_{i}+Y\left(I-\bar{C} \bar{C}^{\dagger}\right) \eta_{1} A_{i}-W_{i} C\right]\right\}, \\
& \tilde{\lambda}_{21}=\left[\alpha_{2} G \bar{C} \eta_{1} D_{i} H_{w}+Y\left(I-\bar{C} \bar{C}^{\dagger}\right) \eta_{1} D_{i} H_{w}\right]^{T}, \\
& \tilde{\lambda}_{22}=-H_{w}^{T} D_{i}^{T} D_{i} H_{w}, \tilde{\lambda}_{31}=\left(\alpha_{2} W_{i} E H_{v}\right)^{T}, \tilde{\lambda}_{33}=-H_{v}^{T} E^{T} E H_{v}, \\
& \tilde{\lambda}_{41}=\left[\alpha_{2} G \bar{C} \eta_{2} E H_{v}+Y\left(I_{n_{x}+n_{y}}-\bar{C} \bar{C}^{\dagger}\right) \eta_{2} E H_{v}\right]^{T}, \tilde{\lambda}_{44}=-H_{v}^{T} E^{T} E H_{v}, \\
& \tilde{\lambda}_{51}=-\alpha_{2} G^{T}+G \bar{C} \eta_{1} A_{i}+Y\left(I-\bar{C} \bar{C}^{\dagger}\right) \eta_{1} A_{i}-W_{i} C, \\
& \tilde{\lambda}_{52}=G \bar{C} \eta_{1} D_{i} H_{w}+Y\left(I-\bar{C} \bar{C}^{\dagger}\right) \eta_{1} D_{i} H_{w}, \tilde{\lambda}_{53}=W_{i} E H_{v}, \\
& \tilde{\lambda}_{54}=G \bar{C} \eta_{2} E H_{v}+Y\left(I_{n_{x}+n_{y}}-\bar{C} \bar{C}^{\dagger}\right) \eta_{2} E H_{v} .
\end{aligned}
$$

with tunable parameters $\alpha_{1}, \alpha_{2}$ and matrix $V \in \mathbb{R}^{n_{f} \times n_{x}}$.

To maximaze the $H_{-}$index $\beta$ and minimize the $P$-radius of zonotope, the following optimization probelm should be solved:

$$
\max _{\text {s.t. }} \beta+\kappa
$$

If (34) is solvable, then the design parameters of (8) can be obtained from

$$
\begin{gathered}
L_{i}=G^{-1} W_{i}, \quad i=1,2, \cdots, s, \\
T=\bar{C} \eta_{1}+G^{-1} Y\left(I_{n_{x}+n_{y}}-\bar{C} \bar{C}^{\dagger}\right) \eta_{1}, \\
N=\bar{C} \eta_{2}+G^{-1} Y\left(I_{n_{x}+n_{y}}-\bar{C} \bar{C}^{\dagger}\right) \eta_{2} .
\end{gathered}
$$

Proof: For the sake of clarity, we divide the proof into two parts: sufficient $H_{-}$fault sensitivity condition and sufficient stability and disturbance attenuation condition.

A. Fault sensitivity condition

The interested frequency range is $\vartheta_{1} \sim \vartheta_{2}$, according to Lemma 2.3, subsystem (16) satisfies (19), i.e. error system (14) has a finite-frequency $H_{-}$performance index $\beta$, if inequality (25) is satisfied. 
Substitute

$$
\Pi=\left[\begin{array}{cc}
-I & 0 \\
0 & \beta^{2} I
\end{array}\right], \Xi=\left[\begin{array}{cc}
-P_{f}\left(h^{+}\right) & \mathrm{e}^{j \vartheta_{c}} Q \\
\mathrm{e}^{-j \vartheta_{c}} Q & P_{f}(h)-2 \cos \vartheta_{w} Q
\end{array}\right]
$$

into (25), then inequality (25) becomes

$$
\left[\begin{array}{cc}
\Phi_{11} & \star \\
\Phi_{21} & \Phi_{22}
\end{array}\right]<0
$$

where

$$
\begin{aligned}
\Phi_{11} & =-[T A(h)-L(h) C]^{T} P_{f}\left(h^{+}\right)[T A(h)-L(h) C]+\mathrm{e}^{-j \vartheta_{c}} Q[T A(h)-L(h) C] \\
& +[T A(h)-L(h) C]^{T} \mathrm{e}^{j \vartheta_{c}} Q+P_{f}(h)-2 \cos \vartheta_{w} Q-C^{T} C, \\
\Phi_{21} & =-[T F(h)]^{T} P_{f}\left(h^{+}\right)[T A(h)-L(h) C]+[T F(h)]^{T} \mathrm{e}^{j \vartheta_{c}} Q, \\
\Phi_{22} & =-[T F(h)]^{T} P_{f}\left(h^{+}\right)[T F(h)]+\beta^{2} I .
\end{aligned}
$$

Note that it is not a trivial work to solve (39) due to the existence of equation constraint (10) and couplings between $L(h)$ and $P_{f}\left(h^{+}\right)$. To facilitate the design, we transform inequalities (39) into linear matrix inequalities. First, we rewrite (39) as

$$
\Theta_{f}+\mathscr{A}_{f}^{T} \mathrm{e}^{j \vartheta_{c}} \mathscr{Q}_{f}^{T}+\mathrm{e}^{-j \vartheta_{c}} \mathscr{Q}_{f} \mathscr{A}_{f}-\mathscr{A}_{f}^{T} P_{f}\left(h^{+}\right) \mathscr{A}_{f}<0,
$$

where

$$
\Theta_{f}=\left[\begin{array}{cc}
P_{f}(h)-2 \cos \vartheta_{w} Q-C^{T} C & \star \\
0 & \beta^{2} I
\end{array}\right], \mathscr{A}_{f}=\left[\begin{array}{cc}
T A(h)-L(h) C & T F(h)
\end{array}\right], \mathscr{Q}_{f}=\left[\begin{array}{l}
Q \\
0
\end{array}\right],
$$

then, it follows

$$
\left[\begin{array}{ll}
I & \mathscr{A}_{f}^{T}
\end{array}\right]\left[\begin{array}{cc}
\Theta_{f} & \mathrm{e}^{-j \vartheta_{c}} \mathscr{Q}_{f} \\
\mathrm{e}^{j \vartheta_{c}} \mathscr{Q}_{f}^{T} & -P_{f}\left(h^{+}\right)
\end{array}\right]\left[\begin{array}{c}
I \\
\mathscr{A}_{f}
\end{array}\right]<0
$$

By defining

$$
\mathscr{U}_{f}=\left[\begin{array}{c}
\mathscr{A}_{f}^{T} \\
-I
\end{array}\right], \mathscr{U}_{f}^{\perp}=\left[\begin{array}{ll}
I & \mathscr{A}_{f}^{T}
\end{array}\right]
$$

and according to Lemma 2.1, a sufficient and necessary condition of (41) is that there exists a matrix $\mathscr{Y}_{f}$ such that

$$
\left[\begin{array}{cc}
\Theta_{f} & \mathrm{e}^{-j \vartheta_{c}} \mathscr{Q}_{f} \\
\mathrm{e}^{j \vartheta_{c}} \mathscr{Q}_{f}^{T} & -P_{f}
\end{array}\right]+\mathscr{U}_{f} \mathscr{Y}_{f}+\mathscr{Y}_{f}^{T} \mathscr{U}_{f}^{T}<0 .
$$


To remove the couplings among matrix $\mathscr{Y}_{f}$, we define

$$
\mathscr{Y}_{f}=\left[\begin{array}{lll}
\alpha_{1} G^{T} & (V G)^{T} & G^{T}
\end{array}\right]
$$

By substituting (42) and (44) into (43), we have

$$
\left[\begin{array}{ccc}
\Omega_{11} & \star & \star \\
\Omega_{21} & \Omega_{22} & \star \\
\Omega_{31} & \Omega_{32} & \Omega_{33}
\end{array}\right]<0,
$$

where

$$
\begin{aligned}
& \Omega_{11}=P_{f}(h)-2 \cos \vartheta_{w} Q-C^{T} C+\mathbf{H e}\left\{\alpha_{1} G[T A(h)-L(h) C]\right\}, \\
& \Omega_{21}=V G[T A(h)-L(h) C]+\left\{\alpha_{1} G T F(h)\right\}^{T}, \\
& \Omega_{22}=\beta^{2} I+\mathbf{H e}\{V G T F(h)\} \\
& \Omega_{31}=\mathrm{e}^{j \vartheta_{c}} Q-\alpha_{1} G^{T}+G[T A(h)-L(h) C], \\
& \Omega_{32}=-(V G)^{T}+G T F(h), \\
& \Omega_{33}=-P_{f}\left(h^{+}\right)-G-G^{T} .
\end{aligned}
$$

By letting $W(h)=G L(h)$ and combining (6) and (9), according to Tanaka \& Wang (2004), the condition (45) is equivalent to (32).

B. Disturbance attenuation condition

In order to achieve a good accuracy, the method presented in Le et al. (2013b) is adopted here. The main idea is to compute a matrix $P_{d}(h)=P_{d}(h)^{T}>0$ such that the $P$-radius of zonotope (22) is minimized.

The guaranteed state estimation at time instant $k$ is the zonotope $\mathcal{X}_{k}$, and its $P$-radius is denoted by $R_{P_{k}}$. A criterion is established as follows:

$$
R_{P_{k+1}}<\gamma R_{P_{k}}+\epsilon, \quad \text { with } \gamma \in(0,1),
$$

where $\epsilon$ is a positive constant, which permits one to bound the influence of disturbance $w_{k}$ and measurement noises $v_{k}$ and $v_{k+1}$, as follows

$$
\epsilon=\max _{b_{1} \in \mathbf{B}^{n_{w}}}\left\|D(h) H_{w} b_{1}\right\|^{2}+\max _{b_{2} \in \mathbf{B}^{n_{v}}}\left\|E H_{v} b_{2}\right\|^{2}+\max _{b_{3} \in \mathbf{B}^{n_{v}}}\left\|E H_{v} b_{3}\right\|^{2} .
$$


where $b_{1} \in \mathbb{R}^{n_{w}}, b_{2} \in \mathbb{R}^{n_{v}}$ and $b_{3} \in \mathbb{R}^{n_{v}}$ are arbitrary vectors which belong to hypercube $\mathbf{B}^{n_{w}}, \mathbf{B}^{n_{v}}$ and $\mathbf{B}^{n_{v}}$, respectively.

Substituting (47) into (46), we have

$$
\begin{array}{r}
\max _{\hat{b} \in \mathbf{B}^{n_{x}+n_{w}+2 n_{v}}}\left\|H_{k+1} \hat{b}\right\|_{P}^{2}<\gamma \max _{b \in \mathbf{B}^{n_{x}}}\left\|H_{k} b\right\|_{P}^{2}+\max _{b_{1} \in \mathbf{B}^{n_{w}}}\left\|D(h) H_{w} b_{1}\right\|^{2}+\max _{b_{2} \in \mathbf{B}^{n_{v}}}\left\|E H_{v} b_{2}\right\|^{2} \\
+\max _{b_{3} \in \mathbf{B}^{n_{v}}}\left\|E H_{v} b_{3}\right\|^{2} .
\end{array}
$$

For all $\hat{b}, b, b_{1}, b_{2}$ and $b_{3},(48)$ is implied by

$$
\left\|H_{k+1} \hat{b}\right\|_{P}^{2}<\gamma\left\|H_{k} b\right\|_{P}^{2}+\left\|D(h) H_{w} b_{1}\right\|^{2}+\left\|E H_{v} b_{2}\right\|^{2}+\left\|E H_{v} b_{3}\right\|^{2} .
$$

Considering $\theta=H_{k} b$ and substituting (22) into (49), we have

$$
\left[\begin{array}{c}
\theta \\
b_{1} \\
b_{2} \\
b_{3}
\end{array}\right]^{T} \mathscr{A}_{d}^{T} P_{d}\left(h^{+}\right) \mathscr{A}_{d}\left[\begin{array}{c}
\theta \\
b_{1} \\
b_{2} \\
b_{3}
\end{array}\right]-\left[\begin{array}{c}
\theta \\
b_{1} \\
b_{2} \\
b_{3}
\end{array}\right]^{T} \Omega\left[\begin{array}{c}
\theta \\
b_{1} \\
b_{2} \\
b_{3}
\end{array}\right]<0
$$

where

$$
\begin{aligned}
& \mathscr{A}_{d}=\left[\begin{array}{llll}
T A(h)-L(h) C & T D(h) H_{w} & L E H_{v} & N E H_{v}
\end{array}\right], \\
& \Omega=\left[\begin{array}{cccc}
\gamma P(h) & 0 & 0 & 0 \\
\star & H_{w}^{T} D^{T}(h) D(h) H_{w} & 0 & 0 \\
\star & \star & H_{v}^{T} E^{T} E H_{v} & 0 \\
\star & \star & \star & H_{v}^{T} E^{T} E H_{v}
\end{array}\right] .
\end{aligned}
$$

Then, we have

$$
\left[\begin{array}{cccc}
\Psi_{11} & \star & \star & \star \\
\Psi_{21} & \Psi_{22} & \star & \star \\
\Psi_{31} & \Psi_{32} & \Psi_{33} & \star \\
\Psi_{41} & \Psi_{42} & \Psi_{43} & \Psi_{44}
\end{array}\right]<0
$$


where

$$
\begin{aligned}
& \Psi_{11}=[T A(h)-L(h) C]^{T} P_{d}\left(h^{+}\right)[T A(h)-L(h) C]-\gamma P_{d}(h), \\
& \Psi_{21}=\left[T D(h) H_{w}\right]^{T} P_{d}\left(h^{+}\right)[T A(h)-L(h) C], \\
& \Psi_{22}=\left[T D(h) H_{w}\right]^{T} P_{d}\left(h^{+}\right)\left[T D(h) H_{w}\right]-H_{w}^{T} D^{T}(h) D(h) H_{w}, \\
& \Psi_{31}=\left(L E H_{v}\right)^{T} P_{d}\left(h^{+}\right)[T A(h)-L(h) C], \\
& \Psi_{32}=\left(L E H_{v}\right)^{T} P_{d}\left(h^{+}\right)\left[T D(h) H_{w}\right], \Psi_{33}=\left(L E H_{v}\right)^{T} P_{d}\left(h^{+}\right) L E H_{v}-H_{v}^{T} E^{T} E H_{v}, \\
& \Psi_{41}=\left(N E H_{v}\right)^{T} P_{d}\left(h^{+}\right)[T A(h)-L(h) C], \Psi_{42}=\left(N E H_{v}\right)^{T} P_{d}\left(h^{+}\right)\left[T D H_{w}\right], \\
& \Psi_{43}=\left(N E H_{v}\right)^{T} P_{d}\left(h^{+}\right)\left(L E H_{v}\right), \Psi_{44}=\left(N E H_{v}\right)^{T} P_{d}\left(h^{+}\right)\left(N E H_{v}\right)-H_{v}^{T} E^{T} E H_{v} .
\end{aligned}
$$

Similarly, rewrite (51) as

$$
\Theta_{d}+\mathscr{A}_{d}^{T} P_{d}\left(h^{+}\right) \mathscr{A}_{d}<0,
$$

where

$$
\begin{gathered}
\Theta_{d}=\left[\begin{array}{cccc}
-\gamma P_{d}(h) & \star & \star & \star \\
0 & -H_{w}^{T} D^{T}(h) D(h) H_{w} & \star & \star \\
0 & 0 & -H_{v}^{T} E^{T} E H_{v} & \star \\
0 & 0 & 0 & -H_{v}^{T} E^{T} E H_{v}
\end{array}\right], \\
\mathscr{A}_{d}=\left[\begin{array}{llll}
T A(h)-L(h) C & T D(h) H_{w} & L E H_{v} & N E H_{v}
\end{array}\right],
\end{gathered}
$$

and further rewrite as

$$
\left[\begin{array}{ll}
I & \mathscr{A}_{d}^{T}
\end{array}\right]\left[\begin{array}{cc}
\Theta_{d} & 0 \\
0 & P_{d}\left(h^{+}\right)
\end{array}\right]\left[\begin{array}{c}
I \\
\mathscr{A}_{d}
\end{array}\right]<0 .
$$

Define

$$
\mathscr{U}_{d}=\left[\begin{array}{c}
\mathscr{A}_{d}^{T} \\
-I
\end{array}\right], \mathscr{U}_{d}^{\perp}=\left[\begin{array}{ll}
I & \mathscr{A}_{d}^{T}
\end{array}\right],
$$

according to Lemma 2.1, (53) equals to

$$
\left[\begin{array}{cc}
\Theta_{d} & 0 \\
0 & P_{d}\left(h^{+}\right)
\end{array}\right]+\mathscr{U}_{d} \mathscr{Y}_{d}+\mathscr{Y}_{d}^{T} \mathscr{U}_{d}^{T}<0 .
$$

By choosing

$$
\mathscr{Y}_{d}=\left[\begin{array}{lllll}
\alpha_{2} G^{T} & 0 & 0 & 0 & G^{T}
\end{array}\right]
$$


and substituting (54) and (56) into (55), we have

$$
\left[\begin{array}{ccccc}
\Gamma_{11} & \star & \star & \star & \star \\
\Gamma_{21} & \Gamma_{22} & \star & \star & \star \\
\Gamma_{31} & 0 & \Gamma_{33} & \star & \star \\
\Gamma_{41} & 0 & 0 & \Gamma_{44} & \star \\
\Gamma_{51} & \Gamma_{52} & \Gamma_{53} & \Gamma_{54} & \Gamma_{55}
\end{array}\right]<0
$$

where

$$
\begin{aligned}
& \Gamma_{11}=-\gamma P_{d}(h)+\mathbf{H e}\left\{\alpha_{2} G[T A(h)-L(h) C]\right\}, \Gamma_{21}=\left(\alpha_{2} G T D(h) H_{w}\right)^{T}, \\
& \Gamma_{22}=-H_{w}^{T} D^{T}(h) D(h) H_{w}, \Gamma_{31}=\left(\alpha_{2} G L(h) E H_{v}\right)^{T}, \Gamma_{33}=-H_{v}^{T} E^{T} E H_{v}, \\
& \Gamma_{41}=\left(\alpha_{2} G N E H_{v}\right)^{T}, \Gamma_{44}=-H_{v}^{T} E^{T} E H_{v}, \Gamma_{51}=-\alpha_{2} G^{T}+G[T A(h)-L(h) C], \\
& \Gamma_{52}=G T D(h) H_{w}, \Gamma_{53}=G L(h) E H_{v}, \Gamma_{54}=G N E H_{v}, \Gamma_{55}=P_{d}\left(h^{+}\right)-G-G^{T} .
\end{aligned}
$$

By letting $W(h)=G L(h)$ and combining the definitions in (6) and (9), according to Tanaka \& Wang (2004), the condition (57) is equivalent to (33).

At infinity, condition (46) becomes $R_{P_{\infty}}=\gamma R_{P_{\infty}}+\epsilon$, then, we have $R_{P_{\infty}}=\epsilon /(1-\gamma)$. Considering an ellipsoid $\mathcal{E}=\left\{x: x^{T} P_{d}(h) x \leq \epsilon /(1-\gamma)\right\}$ which can be normalized to $\mathcal{E}=\{x$ : $\left.x^{T} P_{d}(h) \frac{(1-\gamma)}{\epsilon} x \leq 1\right\}$. This ellipsoid is related to the $P$-radius of the zonotopic guaranted state esimation at infinity. To minimize the $P$-radius of the zonotope, we can find the ellipsoid of the smallest diameter (Boyd et al., 1994). More specifically, to find a matrix $P_{d}(h)=P_{d}(h)^{T}>0 \in \mathbb{R}^{n_{x} \times n_{x}}$ and a constant $\psi$ such that

$$
\max _{\psi, P_{d}(h), \gamma} \psi \quad \text { subject to } \quad \frac{P_{d}(h)(1-\gamma)}{\epsilon} \geq \psi I_{n_{x}}, \psi>0
$$

Herein, we define $\kappa=\frac{\psi \epsilon}{1-\gamma}$, then (58) can be derived from (31).

Remark 3.1: We would like to emphasize that, in practice, observer (8) should be implemented as follows:

$$
\left\{\begin{array}{l}
\chi_{k}=T A\left(h_{-}\right) \hat{x}_{k-1}+T B\left(h_{-}\right) u_{k-1}+L\left(h_{-}\right)\left(y_{k-1}-C \hat{x}_{k-1}\right), \\
\hat{x}_{k}=\chi_{k}+N y_{k} \\
r_{k}=y_{k}-C \hat{x}_{k}
\end{array}\right.
$$

Herein, $h_{k-1}$ denotes the weighting function at time instant $k-1$. As $y_{k}, y_{k-1}$ and $h_{k-1}$ are all available, observer (8) is feasible. In other words, the time instant $k+1$ used in theoretical analysis 
is actually $k$ in the implementation. Moreover, we would like to emphasize that the computational load of parameters $T, L_{i}, i=1,2, \cdots, s$ and $N$ are zero, since they are computed off-line. Thus, the computational load of observer consists of two parts. First, since $A\left(h_{-}\right)=\sum_{i=1}^{s} h_{i}\left(\xi_{k-1}\right) A_{i}, B\left(h_{-}\right)=$ $\sum_{i=1}^{s} h_{i}\left(\xi_{k-1}\right) B_{i}$ and $L\left(h_{-}\right)=\sum_{i=1}^{s} h_{i}\left(\xi_{k-1}\right) L_{i}$, the computational complexity of $A\left(h_{-}\right), B\left(h_{-}\right)$and $L\left(h_{-}\right)$is $(2 s-1)\left(n_{x}^{2}+n_{x} n_{u}+n_{x} n_{y}\right)$. Second, as $A\left(h_{-}\right), B\left(h_{-}\right), L\left(h_{-}\right), y_{k-1}, \hat{x}_{k-1}, u_{k-1} y_{k}$ and $\hat{x}_{k}$ are available, the computational complexity of $r_{k}$ is $n_{x}\left(6 n_{x}+2 n_{u}+2 n_{y}-2\right)+n_{y}\left(2 n_{x}-1\right)+5 n_{y} n_{x}$. Therefore, the computational load of the proposed observer is $O\left(n_{x}^{2}\right)$, which is not very high.

\subsection{Zonotopic fault detection decision scheme}

Combining (14) and (24), we have that when fault free case $0 \in \mathcal{R}_{k}, \forall k \in \mathbb{N}_{k}$ holds. When fault occurs, the center $p_{k}^{r}$ of zonotope $\mathcal{R}_{k}$ will deviate, and condition $0 \in \mathcal{R}_{k}, \forall k \in \mathbb{N}_{k}$ will not be guaranteed. Thus, we develop the following fault detection logic

$$
\begin{cases}0 \in \mathcal{R}_{k} & \text { fault }- \text { free } \\ 0 \notin \mathcal{R}_{k} & \text { fault - alarm. }\end{cases}
$$

As mentioned in Wang et al. (2017a), to implement this logic, the residual zonotope can be characterized in a halfspace representation. Therefore, the zonotopic set contains a sequence of linear constraints, which can be formulated as $\mathcal{R}_{k}=\left\{r_{k} \in \mathbb{R}^{n_{y}} \mid \Sigma r_{k} \leq \varrho\right\}$, where $\Sigma$ and $\varrho$ denote a matrix and a vector from the halfspace representation of the residual zonotope. Therefore, this logic involves solving a constraint satisfaction problem. If the problem is feasible, then the origin of the coordinate is included in the residual zonotope. Otherwise, it is not included.

Remark 3.2: The overall block diagram for the proposed zonotopic fault detection approach is depicted in Figure 3.2. Note that the generated envelop of residual zonotopes can be directly applied to residual evaluation. Thus, compared with the conventional fault detection methods Li et al. (2015); Chen et al. (2015); Zhou et al. (2017); Chadli et al. (2013), the main advantage of the proposed method lies in that it gets rid of the difficulty of designing extra residual evaluation function or threshold generator. 


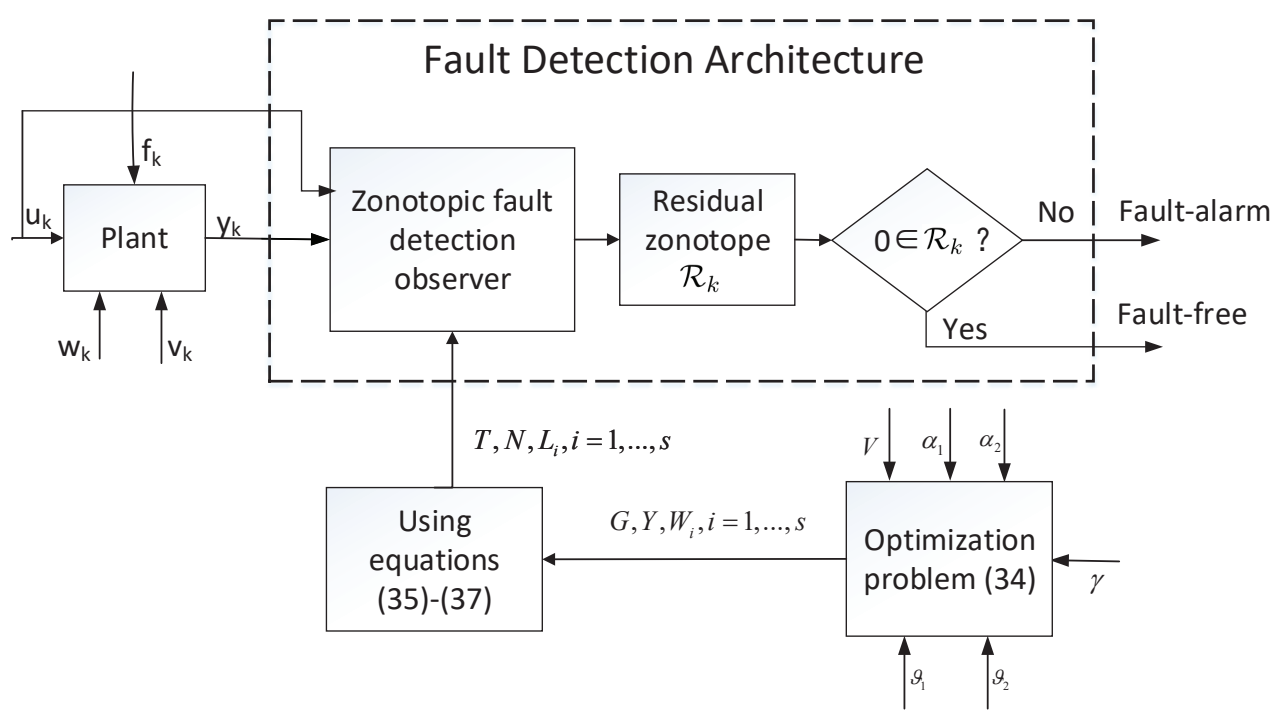

Figure 1: The block diagram of the proposed fault detection approach

\section{Simulation results}

In this section, two examples are simulated to illustrate the performance of the proposed method.

\subsection{Example 1}

To demonstrate the superiority of the proposed method, a benchmark from Zhang \& Yang (2017) is considered.

$$
\left\{\begin{array}{l}
\dot{x}(t)=\left[\begin{array}{cc}
0 & 1 \\
-k & -1
\end{array}\right] x(t)+\left[\begin{array}{l}
0 \\
1
\end{array}\right] w(t)+\left[\begin{array}{l}
1 \\
1
\end{array}\right] f(t), \\
y(t)=\left[\begin{array}{ll}
1 & 0
\end{array}\right] x(t)
\end{array}\right.
$$

Consider $k=k_{0}(1+\xi)$ with $k_{0}=1$ and $|\xi| \leq 1$. By applying the Euler's discretization method with fixed step $T=0.1$ s, the system (60) can be modeled as follows

Rule 1: IF $\xi_{k}$ is about 1, THEN

$$
\left\{\begin{array}{l}
x_{k+1}=A_{1} x_{k}+D_{1} w_{k}+F_{1} f_{k} \\
y_{k}=C x_{k}
\end{array},\right.
$$


Rule 2: IF $\xi_{k}$ is about -1 , THEN

$$
\left\{\begin{array}{l}
x_{k+1}=A_{2} x_{k}+D_{2} w_{k}+F_{2} f_{k} \\
y_{k}=C x_{k}
\end{array},\right.
$$

where

$$
A_{1}=\left[\begin{array}{cc}
1 & 0.1 \\
-0.2 & 0.9
\end{array}\right], A_{2}=\left[\begin{array}{ll}
1 & 0.1 \\
0 & 0.9
\end{array}\right], D_{1}=D_{2}=\left[\begin{array}{c}
0 \\
0.1
\end{array}\right], F_{1}=F_{2}=\left[\begin{array}{l}
0.1 \\
0.1
\end{array}\right], C=\left[\begin{array}{ll}
1 & 0
\end{array}\right],
$$

and the membership functions $h_{1}\left(\xi_{k}\right)$ and $h_{2}\left(\xi_{k}\right)$ are

$$
h_{1}\left(\xi_{k}\right)=\frac{1+\xi_{k}}{2}, \quad h_{2}\left(\xi_{k}\right)=\frac{1-\xi_{k}}{2} .
$$

The term of disturbance used in Zhang \& Yang (2017) is $0.2+0.1|\cos (0.05 k)|$. For comparison, the initial state and the uncertainty are assumed to be bounded by zonotope $\mathcal{X}=\left\langle P_{0}, H_{0}\right\rangle$ and zonotope $\mathcal{W}=\left\langle P_{w}, H_{w}\right\rangle$, respectively, with

$$
P_{0}=\left[\begin{array}{l}
0 \\
0
\end{array}\right], H_{0}=\left[\begin{array}{cc}
0.01 & 0 \\
0 & 0.01
\end{array}\right], P_{w}=0.25, H_{w}=0.05
$$

The interested frequency range in fault sensitivity analysis is chosen as $\theta_{f} \in[0,0.1]$, by setting

$$
\gamma=0.7, \alpha_{1}=-0.9, \alpha_{2}=0.3, V=-8 F_{1}^{T}
$$

and solving the optimization problem (34). Then, we have $\kappa=0.1339, \beta=0.6857$ with the following parameters

$$
L_{1}=\left[\begin{array}{c}
6.5619 \\
-7.0417
\end{array}\right], L_{2}=\left[\begin{array}{c}
6.5619 \\
-6.8417
\end{array}\right], T=\left[\begin{array}{cc}
6.6947 & 0 \\
-6.5579 & 1.0000
\end{array}\right], N=\left[\begin{array}{c}
-5.6947 \\
6.5579
\end{array}\right] .
$$

In the simulation, we consider a small additive actuator fault as follows:

$$
f_{k}= \begin{cases}0, & k \leq 100, \\ 0.05, & k \geq 100,\end{cases}
$$

and the premise variable is borrowed from Zhang \& Yang (2017) that $\xi=0.5$.

The residual obtained under the proposed method and the method of Zhang \& Yang (2017) are depicted in Figure 2. When small fault occurs, it is obvious that the response to fault by the 


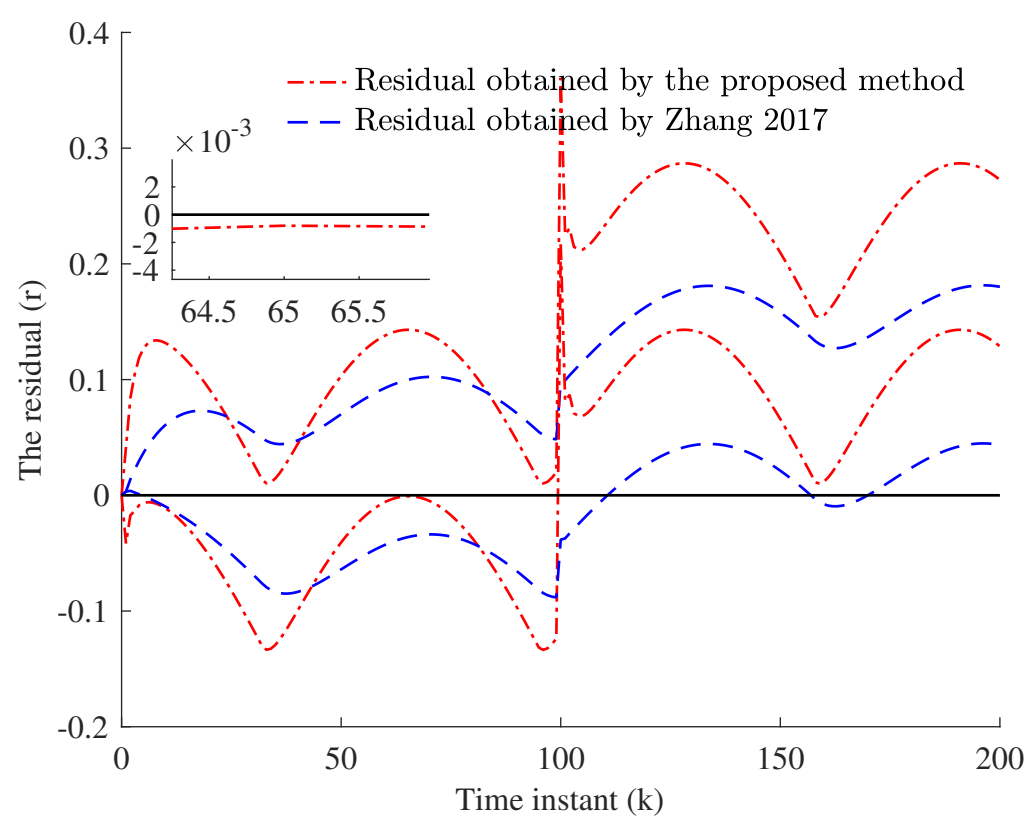

Figure 2: The generated envelop of residual $r_{k}$ by the proposed method and that by Zhang \& Yang (2017)

proposed method is faster than that by Zhang \& Yang (2017), and the relation that $0 \notin \mathcal{R}_{k}$ can not be held always by the method in Zhang \& Yang (2017). From Figure 2, we can also find that the proposed method is more sensitive to fault than that in Zhang \& Yang (2017). The reason is that the frequency characteristics of fault are considered in this paper, while they are not considered in Zhang \& Yang (2017).

\subsection{Example 2}

The proposed method in this paper will be futher validated by the example adopted from Rotondo et al. (2016)

$$
\left\{\begin{array}{l}
x_{k+1}=A\left(\xi_{k}\right) x_{k}+B\left(\xi_{k}\right) u_{k}+F\left(\xi_{k}\right) f_{k}+D\left(\xi_{k}\right) w_{k} \\
y_{k}=C x_{k}+E v_{k}
\end{array}\right.
$$


where

$$
\begin{gathered}
A\left(\xi_{k}\right)=\left[\begin{array}{ccc}
0.3 & 0.2 & \xi_{2_{k}} \\
0.6 & \xi_{1 k} & 0.1 \\
2 \xi_{2 k} & 0.3 & 0.5
\end{array}\right], B\left(\xi_{k}\right)=\left[\begin{array}{cc}
0.8+\xi_{1 k} & 0 \\
0 & 1 \\
0 & 0
\end{array}\right], F\left(\xi_{k}\right)=B\left(\xi_{k}\right), \\
C=\left[\begin{array}{lll}
1 & 0 & 0 \\
0 & 1 & 0
\end{array}\right], D=\left[\begin{array}{ccc}
1 & 0 & 0 \\
0 & 1 & 0 \\
0 & 0 & 1
\end{array}\right], E=\left[\begin{array}{ll}
1 & 0 \\
0 & 1
\end{array}\right] .
\end{gathered}
$$

Since $\xi_{1}, \xi_{2} \in[0.1,0.3]$ for all $k$ instants, the following fuzzy models can be used to model this nonlinear system:

Rule 1: IF $\xi_{1 k}=0.1$ and $\xi_{2 k}=0.1$, THEN

$$
x_{k+1}=A_{1} x_{k}+B_{1} u_{k}+F_{1} f_{k}+D_{1} d_{k}
$$

Rule 2: IF $\xi_{1 k}=0.1$ and $\xi_{2 k}=0.3$, THEN

$$
x_{k+1}=A_{2} x_{k}+B_{2} u_{k}+F_{2} f_{k}+D_{2} d_{k}
$$

Rule 3: IF $\xi_{1 k}=0.3$ and $\xi_{2 k}=0.1$, THEN

$$
x_{k+1}=A_{3} x_{k}+B_{3} u_{k}+F_{3} f_{k}+D_{3} d_{k}
$$

Rule 4: IF $\xi_{1 k}=0.3$ and $\xi_{2 k}=0.3$, THEN

$$
x_{k+1}=A_{4} x_{k}+B_{4} u_{k}+F_{4} f_{k}+D_{4} d_{k} .
$$

In the simulation, the premise variables $\xi_{1 k}$ and $\xi_{2 k}$ are shown in Figure 3 and the membership functions for rules $1,2,3$ and 4 are

$$
\begin{aligned}
& \rho_{1}\left(\xi_{k}\right)=\frac{\left(0.3-\xi_{1 k}\right)\left(0.3-\xi_{2 k}\right)}{0.04}, \rho_{2}\left(\xi_{k}\right)=\frac{\left(0.3-\xi_{1 k}\right)\left(\xi_{2 k}-0.1\right)}{0.04}, \\
& \rho_{3}\left(\xi_{k}\right)=\frac{\left(\xi_{1 k}-0.1\right)\left(0.3-\xi_{2 k}\right)}{0.04}, \rho_{1}\left(\xi_{k}\right)=\frac{\left(\xi_{1 k}-0.1\right)\left(\xi_{2 k}-0.1\right)}{0.04} .
\end{aligned}
$$




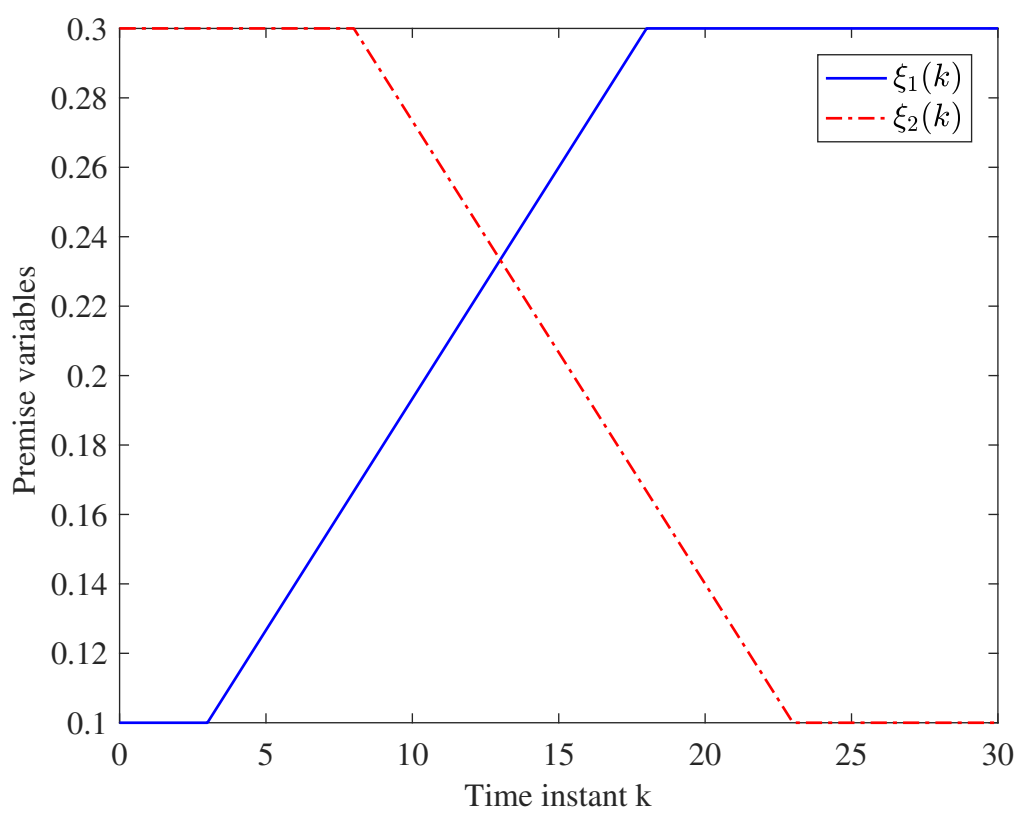

Figure 3: Premise variables

The initial state and the uncertainties are assumed to be bounded by zonotope $\mathcal{X}=\left\langle P_{0}, H_{0}\right\rangle$, zonotope $\mathcal{W}=\left\langle 0, H_{w}\right\rangle$ and zonotope $\mathcal{V}=\left\langle 0, H_{v}\right\rangle$ respectively, with

$$
P_{0}=\left[\begin{array}{l}
0 \\
0 \\
0
\end{array}\right], H_{0}=\left[\begin{array}{ccc}
0.1 & 0 & 0 \\
0 & 0.1 & 0 \\
0 & 0 & 0.1
\end{array}\right], H_{w}=\left[\begin{array}{ccc}
0.02 & 0 & 0 \\
0 & 0.02 & 0 \\
0 & 0 & 0.02
\end{array}\right], H_{v}=\left[\begin{array}{cc}
0.01 & 0 \\
0 & 0.01
\end{array}\right]
$$

The interested frequency range in fault sensitivity analysis is chosen as $\theta_{f} \in[0,0.1]$, by setting

$$
\gamma=0.9, \alpha_{1}=-0.55, \alpha_{2}=-0.9, V=-19 F_{1}^{T}
$$

and solving the optimization problem (34). Then, we have $\kappa=0.0339, \beta=0.5095$ with the following parameters

$$
\begin{gathered}
L_{1}=\left[\begin{array}{cc}
-0.4399 & 0.1864 \\
0.5330 & -0.6834 \\
-0.0241 & 0.1893
\end{array}\right], L_{2}=\left[\begin{array}{cc}
-0.4268 & 0.1921 \\
0.5324 & -0.6852 \\
0.3614 & 0.2021
\end{array}\right], L_{3}=\left[\begin{array}{cc}
-0.4310 & 0.2078 \\
0.5349 & -0.5040 \\
-0.0269 & 0.1605
\end{array}\right], \\
L_{4}=\left[\begin{array}{cc}
-0.4165 & 0.2142 \\
0.5340 & -0.5056 \\
0.3600 & 0.1719
\end{array}\right], T=\left[\begin{array}{ccc}
0.9440 & 0.1049 & 0 \\
-0.0634 & 0.9528 & 0 \\
-0.1928 & -0.1649 & 1.0000
\end{array}\right], N=\left[\begin{array}{cc}
0.0560 & -0.1049 \\
0.0634 & 0.0472 \\
0.1928 & 0.1649
\end{array}\right] .
\end{gathered}
$$


To demostrate the applicability of the proposed method, two fault scenarios are considered:

Scenario 1: abrupt fault

$$
f_{k}= \begin{cases}{\left[\begin{array}{ll}
0 & 0
\end{array}\right]^{T},} & k<16 \\
{\left[\begin{array}{ll}
0.1 & 0.2
\end{array}\right]^{T},} & k \geq 16\end{cases}
$$

Scenario 2: time-varying fault

$$
f_{k}= \begin{cases}{\left[\begin{array}{ll}
0 & 0
\end{array}\right]^{T},} & k<16, \\
{\left[\begin{array}{ll}
0.2+0.1 \sin (k-5) & 0
\end{array}\right]^{T},} & k \geq 16,\end{cases}
$$

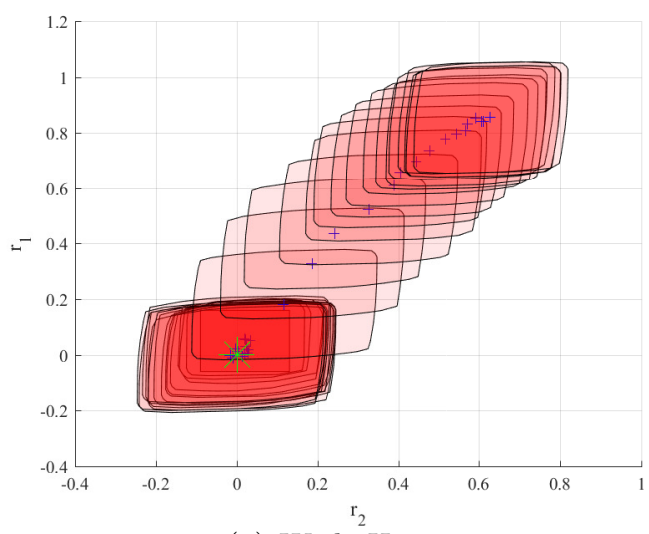

(a) With $H_{-}$

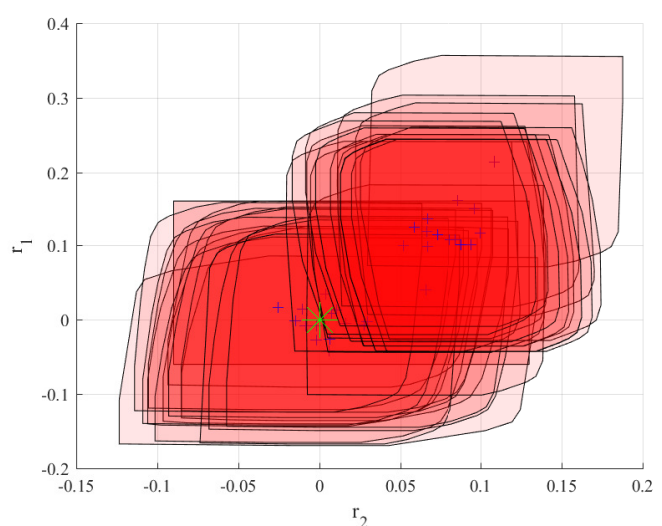

(b) Without $H_{-}$

Figure 4: Comparision of residual zonotopes for abrupt fault

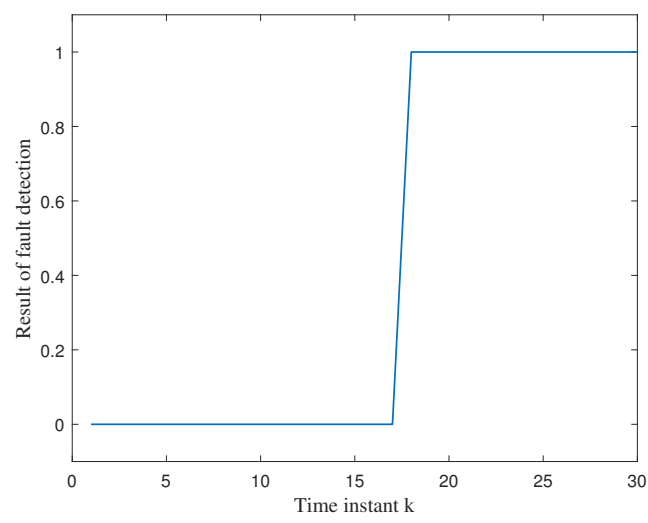

(a) With $H_{-}$

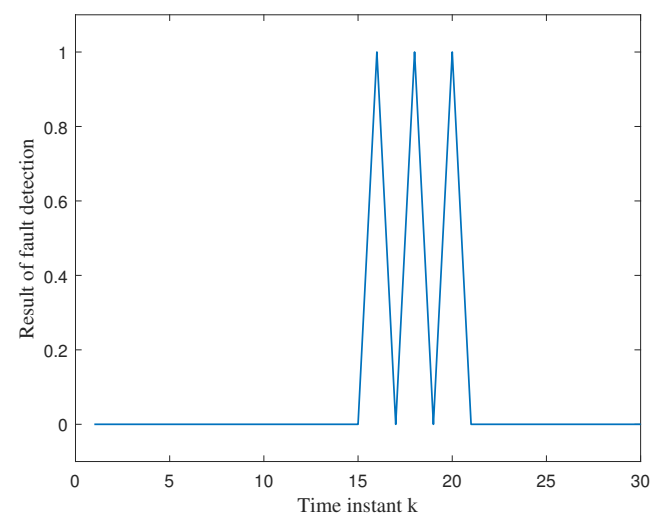

(b) Without $H_{-}$

Figure 5: Comparision of fault detection result for abrupt fault 


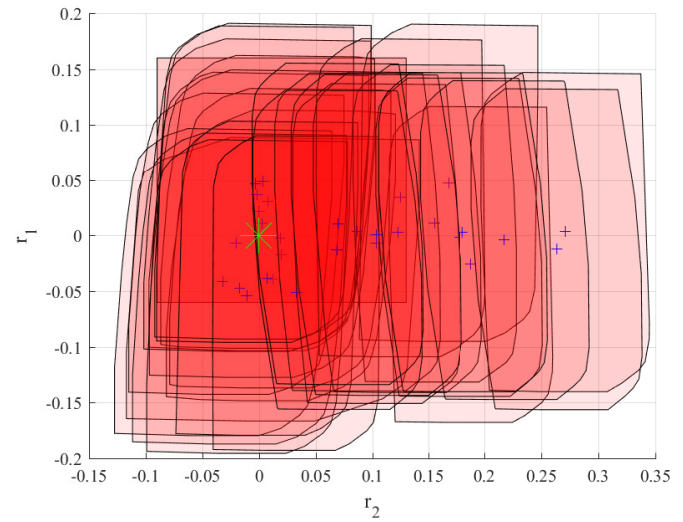

(a) With ${ }^{2}$

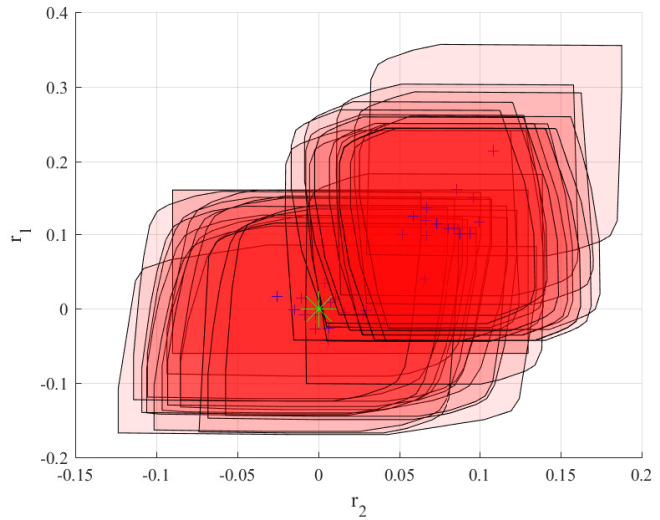

(b) Without $H_{-}$

Figure 6: Comparision of residual zonotopes for time-varying fault

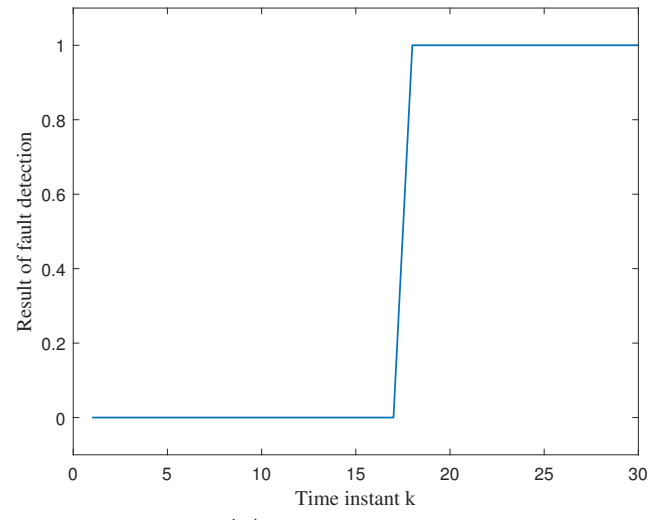

(a) With $H_{-}$

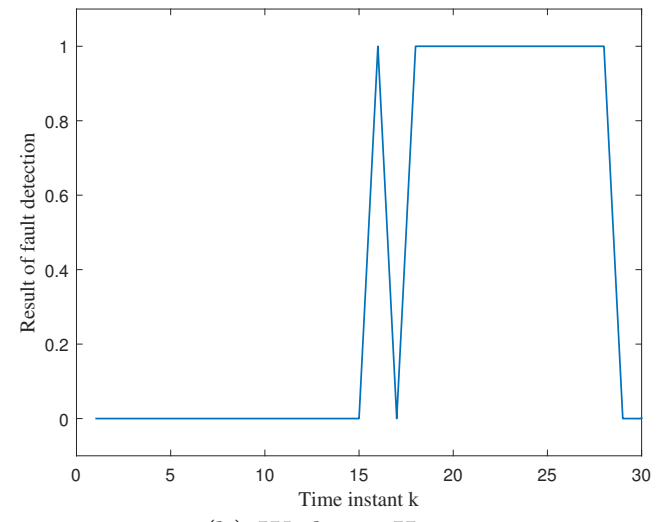

(b) Without $H_{-}$

Figure 7: Comparision of fault detection result for time-varying fault

Table 1: Performance comparison

Theorem 3.1 Full frequency Luenberger form

\begin{tabular}{llll}
\hline$\beta$ & 0.5095 & 0.3453 & 0.4329 \\
$\kappa$ & 0.0339 & 0.0321 & 0.0069 \\
\hline
\end{tabular}


To demonstrate the performance of the proposed method with finite-frequency $H_{-}$index, the standard zonotopic method in Le et al. (2013b) that only consider $P$-radius minimization for state estimation is considered here for comparison. The comparison results are depicted in Figure 4- Figure 7. Herein, the black line represents the zonotopes bounds of residual, the red zone represents the admissible set of residual, the blue plus sign represents the center of corresponding zonotopes, the green asterisk represents the origin of the coordinate. These zonotopes are changed because of the occurrence of faults. Following the decision scheme in Section 3.2, we can detect the fault by checking whether these zonotopes include 0 or not. Herein, we use 0 to denote the case that $0 \in \mathcal{R}_{k}$ and 1 for that $0 \notin \mathcal{R}_{k}$.

From Figure 4 and Figure 6, we can see that the residual zonotopes by using the proposed method are more sensitive to fault than that by using the standard zonotopic method. The fault detection results in Figure. 5 and Figure 7 also show that the proposed method has better fault detection performance.

To further demonstrate the effectiveness of the proposed method, we have added a performance comparison. The results are shown in Table 1. Herein, Theorem 3.1 considers the proposed zonotopic observer design method in finite-frequency domain. Full frequency considers the proposed method applied in full frequency domain. The Luenberger form considers the comonly used Luenberger form observer applied in finite frequency domain. This result can be easily obtained by setting the introduced matrices $T=I$ and $N=0$ in Theorem 3.1. From Table 1, we can conclude that the fault detection performance is improved by considering the frequency characteristics of fault. Furthermore, this performance can be further enhanced by the proposed fault detection method. 


\section{Conclusion}

A zonotopic fault detection observer design method is proposed for discrete-time T-S fuzzy systems. We propose a novel observer structure and develop an design method based on GKYP lemma and $P$-radius minimization approach. The design conditions are expressed in terms of LMIs, which can be efficiently solved. The effectiveness of the proposed method have been illustrated via numerical simulations. In the furture, we can combine the proposed method with unknown input observer to further improve the performance of fault detection. Other potential future work involves applying the proposed method to the detection of actuator fault of vehicle lateral dynamic systems.

\section{Disclosure statement}

No potential conflict of interest was reported by the authors.

\section{Funding}

This work was partially supported by National Natural Science Foundation of China (61773145, 61403104) and the Fundamental Research Funds for the Central Universities (HIT.KLOF.2015.076).

\section{References}

Alamo, T., Bravo, J. M., \& Camacho, E. F. (2005). Guaranteed state estimation by zonotopes. Automatica, 41, $1035-1043$

Basseville, M., Nikiforov, I. V. et al. (1993). Detection of abrupt changes: theory and application volume 104. Prentice Hall Englewood Cliffs.

Boyd, S., El Ghaoui, L., Feron, E., \& Balakrishnan, V. (1994). Linear matrix inequalities in system and control theory. SIAM.

Chadli, M., Abdo, A., \& Ding, S. X. (2013). $H_{-} / H_{\infty}$ fault detection filter design for discrete-time Takagi-Sugeno fuzzy system. Automatica, 49, 1996-2005.

Chadli, M., \& Karimi, H. R. (2013). Robust observer design for unknown inputs Takagi-Sugeno models. IEEE Transactions on Fuzzy Systems, 21, 158-164. 
Chang, W.-J., Tsai, Y.-S., \& Ku, C.-C. (2014). Fuzzy fault tolerant control via Takagi-Sugeno fuzzy models for nonlinear systems with multiplicative noises. International Journal of Fuzzy Systems, 16, 338-349.

Chen, J., Cao, Y.-Y., \& Zhang, W. (2015). A fault detection observer design for LPV systems in finite frequency domain. International Journal of Control, 88, 571-584.

Chen, J., \& Patton, R. J. (2012). Robust model-based fault diagnosis for dynamic systems. Springer Science \& Business Media.

Chibani, A., Chadli, M., \& Braiek, N. B. (2016). A finite frequency approach to $\mathrm{H}_{\infty}$ filtering for T-S fuzzy systems with unknown inputs. Asian Journal of Control, 18, 1608-1618.

Chibani, A., Chadli, M., Shi, P., \& Braiek, N. B. (2017). Fuzzy fault detection filter design for T-S fuzzy systems in the finite-frequency domain. IEEE Transactions on Fuzzy Systems, 25, 1051-1061.

Combastel, C. (2015). Zonotopes and kalman observers: Gain optimality under distinct uncertainty paradigms and robust convergence. Automatica, 55, 265-273.

Ding, D.-W., \& Yang, G.-H. (2010). Fuzzy filter design for nonlinear systems in finite-frequency domain. IEEE Transactions on Fuzzy Systems, 18, 935-945.

Iwasaki, T., \& Hara, S. (2005). Generalized KYP lemma: Unified frequency domain inequalities with design applications. IEEE Transactions on Automatic Control, 50, 41-59.

Iwasaki, T., Hara, S., \& Fradkov, A. L. (2005). Time domain interpretations of frequency domain inequalities on (semi) finite ranges. System \& Control Letters, 54, 681-691.

Khan, A. Q., \& Ding, S. X. (2011). Threshold computation for fault detection in a class of discrete-time nonlinear systems. International Journal of Adaptive Control and Signal Processing, 25, 407-429.

Kurzhanski, A. B., \& Varaiya, P. (2000). Ellipsoidal techniques for reachability analysis: internal approximation. System $\&$ Control Letters, 41, 201-211.

Le, V. T. H., Stoica, C., Alamo, T., Camacho, E. F., \& Dumur, D. (2013a). Zonotopes: From guaranteed stateestimation to control. John Wiley \& Sons.

Le, V. T. H., Stoica, C., Alamo, T., Camacho, E. F., \& Dumur, D. (2013b). Zonotopic guaranteed state estimation for uncertain systems. Automatica, 49, 3418-3424.

Li, X., Liu, H. H., \& Jiang, B. (2015). Fault detection filter design with optimization and partial decoupling. IEEE Transactions on Automatic Control, 60, 1951-1956.

Li, X.-J., \& Yang, G.-H. (2014). Fault detection in finite frequency domain for Takagi-Sugeno fuzzy systems with sensor faults. IEEE Transactions on Cybernetics, 44, 1446-1458. 
Li, X.-J., \& Yang, G.-H. (2015). Fault detection in finite frequency domains for multi-delay uncertain systems with application to ground vehicle. International Journal of Robust and Nonlinear Control, 25, 3780-3798.

Li, Y., Karimi, H. R., Zhong, M., Ding, S. X., \& Liu, S. (2018). Fault detection for linear discrete time-varying systems with multiplicative noise: The finite-horizon case. IEEE Transactions on Circuits and Systems I: Regular Papers, . doi:10.1109/TCSI.2018.2832229.

Liu, J., Wang, J. L., \& Yang, G.-H. (2005). An LMI approach to minimum sensitivity analysis with application to fault detection. Automatica, 41, 1995-2004.

Pan, Y., \& Yang, G.-H. (2017). Fault detection for interval type-2 fuzzy stochastic systems with D stability constraint. International Journal of Systems Science, 48, 43-52.

Puig, V. (2010). Fault diagnosis and fault tolerant control using set-membership approaches: Application to real case studies. International Journal of Applied Mathematics and Computer Science, 20, 619-635.

Raïssi, T., Efimov, D., \& Zolghadri, A. (2012). Interval state estimation for a class of nonlinear systems. IEEE Transactions on Automatic Control, 57, 260-265.

Ren, Y., Ding, D.-W., \& Li, Q. (2017). Finite-frequency fault detection for two-dimensional fornasini-marchesini dynamical systems. International Journal of Systems Science, (pp. 1-12).

Rotondo, D., López-Estrada, F.-R., Nejjari, F., Ponsart, J.-C., Theilliol, D., \& Puig, V. (2016). Actuator multiplicative fault estimation in discrete-time LPV systems using switched observers. Journal of the Franklin Institute, 353, 3176-3191.

Tanaka, K., \& Wang, H. O. (2004). Fuzzy control systems design and analysis: a linear matrix inequality approach. John Wiley \& Sons.

Tornil-Sin, S., Ocampo-Martinez, C., Puig, V., \& Escobet, T. (2014). Robust fault diagnosis of nonlinear systems using interval constraint satisfaction and analytical redundancy relations. IEEE Transactions on Systems, Man, and Cybernetics: Systems, 44, 18-29.

Wang, H., \& Yang, G.-H. (2008). A finite frequency domain approach to fault detection observer design for linear continuous-time systems. Asian Journal of Control, 10, 559-568.

Wang, Y., Puig, V., \& Cembrano, G. (2018). Set-membership approach and Kalman observer based on zonotopes for discrete-time descriptor systems. Automatica, 93, 435-443.

Wang, Y., Zhou, M., Puig, V., Cembrano, G., \& Wang, Z. (2017a). Zonotopic fault detection observer with $H_{-}$ performance. In 36th Chinese Control Conference (CCC) (pp. 7230-7235). IEEE.

Wang, Z., Rodrigues, M., Theilliol, D., \& Shen, Y. (2014). Sensor fault estimation filter design for discrete-time 
linear time-varying systems. Acta Automatica Sinica, 40, 2364-2369.

Wang, Z., Rodrigues, M., Theilliol, D., \& Shen, Y. (2015). Actuator fault estimation observer design for discrete-time linear parameter-varying descriptor systems. International Journal of Adaptive Control and Signal Processing, 29, 242-258.

Wang, Z., Shen, Y., Zhang, X., \& Wang, Q. (2012). Observer design for discrete-time descriptor systems: An LMI approach. System \& Control Letters, 61, 683-687.

Wang, Z., Shi, P., \& Lim, C.-C. (2017b). $H_{-} / H_{\infty}$ fault detection observer in finite frequency domain for linear parameter-varying descriptor systems. Automatica, 86, 38-45.

Xu, F., Puig, V., Ocampo-Martinez, C., Stoican, F., \& Olaru, S. (2014). Actuator-fault detection and isolation based on set-theoretic approaches. Journal of Process Control, 24, 947-956.

Youssef, T., Chadli, M., Karimi, H., \& Wang, R. (2017). Actuator and sensor faults estimation based on proportional integral observer for T-S fuzzy model. Journal of the Franklin Institute, 354, 2524-2542.

Zhai, D., An, L., Dong, J., \& Zhang, Q. (2018). Switched adaptive fuzzy tracking control for a class of switched nonlinear systems under arbitrary switching. IEEE Transactions on Fuzzy Systems, 26, 585-597.

Zhang, K., Jiang, B., Shi, P., \& Xu, J. (2015). Analysis and design of robust $H_{\infty}$ fault estimation observer with finite-frequency specifications for discrete-time fuzzy systems. IEEE Transactions on Cybernetics, 45, 1225-1235.

Zhang, Z.-H., \& Yang, G.-H. (2017). Fault detection for discrete-time LPV systems using interval observers. International Journal of Systems Science, 48, 2921-2935.

Zhou, M., Wang, Z., Shen, Y., \& Shen, M. (2017). $H_{-} / H_{\infty}$ fault detection observer design in finite-frequency domain for lipschitz non-linear systems. IET Control Theory \& Applications, 11, 2361 - 2369.

Zhuang, G., Li, Y., \& Li, Z. (2016). Fault detection for a class of uncertain nonlinear markovian jump stochastic systems with mode-dependent time delays and sensor saturation. International Journal of Systems Science, 47, 1514-1532. 